\title{
Contaminação fúngica e determinação de patulina em maçãs 'Fuji Mishima' sob armazenamento em atmosfera controlada dinâmica
}


Andrieli Stefanello ${ }^{1}$, Fabio Rodrigo Thewes ${ }^{1}$, Suele Fernanda Pregadier Schmidt ${ }^{1}$, Auri Brackmann ${ }^{1}$, Marina Venturini Copetti ${ }^{1}$, Ionara Regina Pizzutti ${ }^{1}$, Roger Wagner ${ }^{1}$

\begin{abstract}
Resumo
O objetivo deste estudo foi avaliar a contaminação de fungos e patulina em maçãs 'Fuji Mishima' sob armazenamento em atmosfera controlada (AC) e atmosfera controlada dinâmica sob quociente respiratório (ACD-QR) de 1,3 combinado a diferentes pCO 2 . As maçãs foram armazenadas oito meses nas condições acima mencionadas, a $0,5^{\circ} \mathrm{C}$ somados a 7 dias de vida de prateleira a 20 ${ }^{\circ} \mathrm{C}$. Fungos toxigênicos e presença de patulina foram encontrados nas maçãs provenientes de todos os tratamentos. AC apresentou a maior concentração de patulina. ACD-QR1,3 + 0,8 kPa CO 2 (menor pCO2) apresentou a menor concentração da toxina, estando abaixo do limite da legislação $\left(50 \mu \mathrm{g} \mathrm{kg}^{-1}\right)$.
\end{abstract}

Palavras-chave: Qualidade de frutas; Micotoxinas; Pós-colheita

\section{Introdução}

A maçã é uma cultura relevante em todo o mundo, com uma produção estimada de 75,8 milhões de toneladas na safra 2019/2020 (FAO, 2020). As indústrias da maçã costumam processar a fruta ao longo do ano, o que requer o armazenamento da fruta por vários meses. Com o objetivo de prevenir a podridão dos frutos, aumentar a vida útil e preservar as características organolépticas, um novo método para armazenamento de maçãs em condições de atmosfera controlada dinâmica (DCA), baseado no quociente respiratório (DCA-RQ) foi recentemente desenvolvido e estudado no Brasil e outros países (BOTH et al., 2017; ANESE et al., 2020). Porém, mesmo em CA e DCA ocorrem perdas durante o armazenamento prolongado, principalmente por ocorrência de podridão (WATKINS, 2010). A podridão do mofo azul causada por Penicillium expansum é uma das principais causas de perdas pós-colheita em maçãs armazenadas (TANNOUS et al., 2018). Durante o crescimento em frutos, $P$. expansum pode produzir patulina, um metabólito secundário com efeitos potenciais mutagênicos, carcinogênicos e teratogênicos em humanos (PUEL et al., 2010). No Brasil, a legislação estabelece um nível máximo de patulina de $50 \mu \mathrm{g}$ $\mathrm{kg}^{-1}$ para sucos e polpas de maçã (ANVISA, 2011).

Mesmo que a prática de processamento de frutas mofadas seja evitada em vários países, frutas com podridão ainda podem ser incorporadas à linha de processamento, principalmente quando a decomposição fúngica é incipiente ou não detectável pelos controles de qualidade padrão realizados pelas indústrias de maçã (PATRIARCA, 2019). Vários estudos relataram que a concentração de patulina nos sucos de maçã e outros subprodutos da maçã ultrapassou os limites estabelecidos pelas agências reguladoras (OTEIZA et al., 2017).
No entanto, há pouca informação sobre a capacidade do $P$. expansum e outros fungos de infectar e produzir patulina em maçãs armazenadas sob ACD-RQ combinado a várias $\mathrm{pCO}_{2}$. Neste estudo, a presença de fungos e a produção de patulina foram investigadas em maçãs 'Fuji Mishima' sob CA e ACD-RQ com diferentes $\mathrm{pCO}_{2}$.

\section{Material e métodos}

Os materiais experimentais consistiram de maçãs 'Fuji Mishima' colhidas em 2018 em pomares comerciais na cidade de Vacaria (RS, Brasil). O experimento foi conduzido em delineamento inteiramente casualizado com quatro repetições por tratamento. Os tratamentos foram compostos por cinco condições de armazenamento: i) Atmosfera controlada (AC) com 1,0 kPa O2 e 1,2 kPa CO2; ii) ACD-QR 1,3 e 0,8 kPa CO2; iii) ACD-QR 1,3 e 1,2 kPa CO2; iv) ACD-QR 1,3 e 1,6 kPa CO2; v) ACD-QR 1,3 e 2.0 kPa CO2. Quatro repetições de 25 frutas foram utilizadas para cada tratamento. As frutas foram armazenadas por oito meses (WEBER et al., 2015).

Após o período de armazenamento os frutos foram estocados mais sete dias de vida de prateleira a $20^{\circ} \mathrm{C}$, e então a incidência e severidade da podridão foram avaliadas a fim de padronizar a amostragem para subsequentes análises de qualidade. A incidência de podridão foi avaliada pela contagem de frutos com lesões fúngicas típicas maiores que $5 \mathrm{~mm}$ de diâmetro, de acordo com Brackmann et al. (2015). A severidade foi avaliada de acordo com os índices de podridão da área dos frutos afetados: índice 1: 0 - 25\% da área superficial dos frutos podres; $2: 26-50 \%$ da área de superfície da fruta podre; 3: 56 - 75\% da área superficial da fruta podre e 4: 76 - $100 \%$ da área superficial da fruta podre. 
A fim de simular maçãs que podem ir para o processamento da indústria, frutas de índice $1(0-25 \%)$ foram escolhidas para realizar as análises de qualidade.

Para identificação fúngica, assepticamente, foram pesados $10 \mathrm{~g}$ de cada amostra de maçã triturada e adicionados $90 \mathrm{~mL}$ de água peptonada $0,1 \%$ e homogeneizados em Smasher (bioMérieux, Brasil), por 1 min. Posteriormente, foram feitas diluições seriadas e alíquotas de $100 \mu \mathrm{L}$ foram inoculadas pela técnica de plaqueamento superficial em placas de Petri contendo $18 \%$ de DG18. Em seguida, as placas foram incubadas a $25^{\circ} \mathrm{C}$ durante 7 dias. Após o período de incubação, as colônias foram contadas e os resultados expressos em logaritmo de unidades formadoras de colônias por grama de amostra (log UFC/g). Por fim, a identificação taxonômica dos gêneros foi realizada em placas contendo meios de cultura específicos para identificação (FRISVAD \& SAMSON, 2004; PITT \& HOCKING, 2009).

A determinação de patulina foi realizada de acordo com Dias et al. (2019). Dessa forma, maçãs foram homogeneizadas em um multiprocessador e $10 \pm 0,1 \mathrm{~g}$ extraídas com acetato de etila (10 $\mathrm{mL}$ ) contendo $30 \mu \mathrm{L}$ de ácido acético, seguido da adição de $4 \mathrm{~g}$ de sulfato de sódio. Agitação mecânica foi realizada por $5 \mathrm{~min}$ e, após centrifugação (4500 rpm por $5 \mathrm{~min}$ ), uma alíquota de 4,0 $\mathrm{mL}$ da camada superior foi pipetada em um tubo contendo $0,6 \mathrm{~g}$ de sulfato de sódio e $1,2 \mathrm{~g}$ de sílica Bondesil $(40 \mu \mathrm{m})$, utilizados para a etapa de purificação. A agitação manual foi realizada por $1 \mathrm{~min}$ e centrifugada a $4500 \mathrm{rpm}$ por $5 \mathrm{~min}$. Uma alíquota de $2,0 \mathrm{~mL}$ foi evaporada a $65{ }^{\circ} \mathrm{C}$ e adicionado $0,5 \mathrm{~mL}$ de solução aquosa de acetonitrila $(25 \%)$ para reconstituir o extrato, o qual foi submetido a UPLC-MS/MS da Waters (Milford, EUA). Uma coluna cromatográfica BEH C18 $(100 \mathrm{~mm} \times 2,1 \mathrm{~mm}$ d.i.; $1,7 \mu \mathrm{m}$ de tamanho de partícula) foi usada a uma temperatura constante de $30^{\circ} \mathrm{C}$. A fase móvel foi água com $2 \mathrm{~g} \mathrm{~L}^{-1}$ de formato de amônio e acetonitrila (80:20 v/v) usando eluição isocrática a uma vazão constante de $0,3 \mathrm{~mL} \mathrm{~min}{ }^{-1}$. Um volume de $10 \mu \mathrm{L}$ de amostra foi injetado no sistema cromatográfico.

Por fim, os dados foram submetidos à análise de variância (ANOVA) e as médias dos tratamentos comparadas pelo teste de Tukey $(p<0,05)$. Essa análise foi realizada por meio do software Statistica 7.0 (StatSoft Inc., Tulsa, OK, EUA).

\section{Resultados e discussão}

Com relação à identificação de contaminação fúngica, um total de 6 gêneros e 3 espécies de fungos filamentosos foram identificados nas amostras de maçãs infectadas após oito meses de armazenamento em AC e ACD somados a sete dias de vida de prateleira a $20^{\circ} \mathrm{C}$. P. expansum foi a espécie mais prevalente $(73 \%)$. A Figura 1a mostra a concentração de fungos encontrada em cada uma das condições de AC e ACD estudadas. Nenhuma diferença significativa foi encontrada entre os tratamentos aplicados na estocagem das maçãs. Esse resultado era esperado pois todas as amostras estão na mesma escala de severidade (até 25\%). Comparando apenas Penicillium sp incluindo a presença de $P$. expansum nas amostras também não foram encontradas diferenças entre os tratamentos (Fig. 1b).

Todas as maçãs infectadas (até $25 \%$ ACD condições de armazenamento mais sete dias de vida de prateleira a $20{ }^{\circ} \mathrm{C}$.

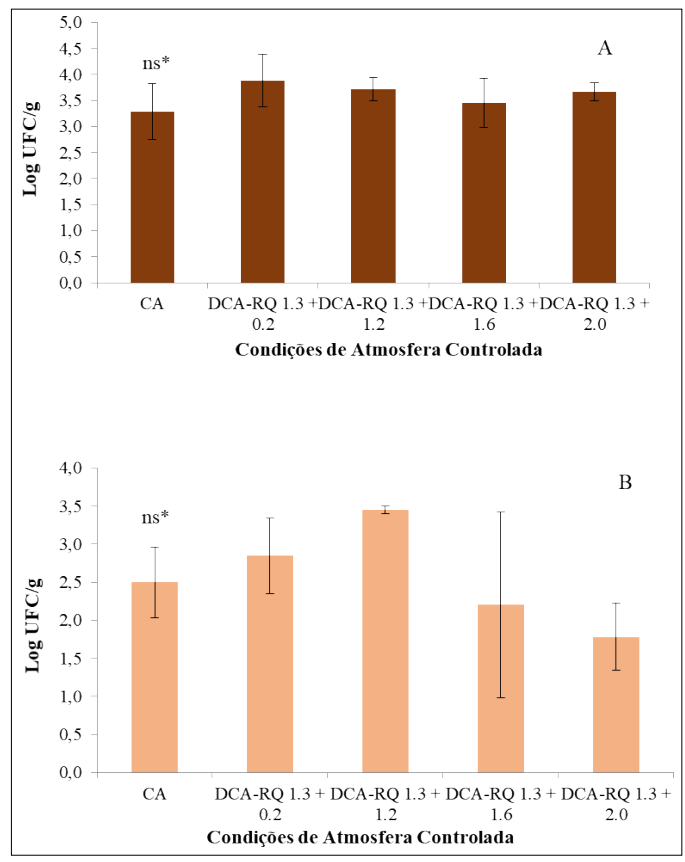

Figura 1: Contaminação fúngica (Log CFU/g) em maçãs 'Fuji Mishima' (a). Penicillium sp. incluindo contaminação por $P$. expansum (Log CFU/g) em maçãs 'Fuji Mishima'. ns: não significativo (b). AC: atmosfera controlada;ACD$\mathrm{QR}+\mathrm{kPa} \mathrm{CO}$ : atmosfera controlada dinâmica com quociente respiratório.

Segundo Patriarca (2019) vários patógenos, que já colonizaram o interior da maçã, são capazes de desenvolver sintomas em atmosfera controlada e, em alguns casos, mesmo assim sintetizam micotoxinas durante o armazenamento. Essa afirmação concorda com nosso estudo, pois em $100 \%$ das amostras foi possível detectar a presença de patulina. Para todas as condições de armazenamento de maçãs 'Fuji Mishima', as concentrações de patulina ficaram acima do nível máximo permitido $\left(50 \mu \mathrm{g} \mathrm{kg}^{-1}\right.$, ANVISA, 2011) com exceção de DCA-RQ1.3 + 0,8 kPa CO 2 . CA mostrou a maior concentração de patulina em comparação com todas as condições de DCA (166 $\mu \mathrm{g} \mathrm{kg}^{-1}$ ) (Fig. 2).

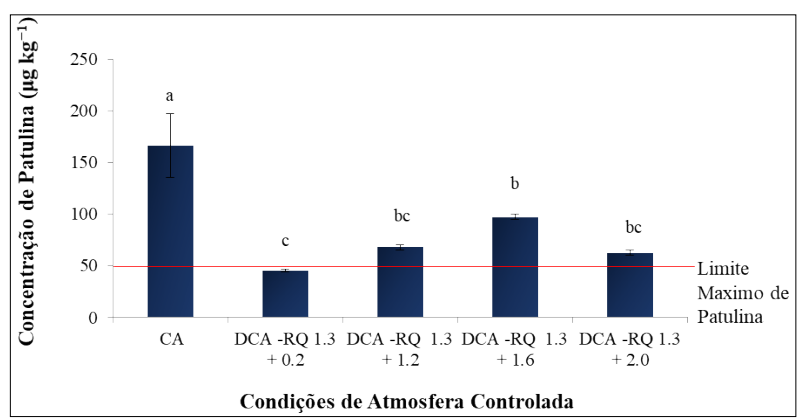

Figura 2: Concentração de patulina de maçãs 'Fuji Mishima' após oito meses de condições de armazenamento em atmosfera controlada mais sete dias a $20^{\circ} \mathrm{C}$. *Médias seguidas de letras diferentes nas colunas diferem pelo teste de Tukey, a $5 \%$ de probabilidade $(p<0,05)$; CA: atmosfera controlada; ACD$\mathrm{QR}+\mathrm{kPa} \mathrm{CO}_{2}$ : atmosfera dinâmica controlada com quociente respiratório.

Considerando as concentrações de fungo e patulina, não houve correlação entre o aumento da patulina e Penicillium sp. mais $P$. expansum para maçãs Fuji Mishima $\left(r^{2}=0,0233\right)$. Nesse sentido, 
essa não correlação sugere que mesmo em amostras com baixa contaminação de fungos, altas concentrações dessa micotoxina podem ser detectadas.

\section{Conclusão}

Mesmo usando tecnologias modernas de armazenamento, espécies toxigênicas e patulina foram encontradas em maçãs

\section{Referências}

ANESE, R. de O.; THEWES, F. R.; BRACKMANN, A.; SCHULTZ, E. E.; WAGNER, R.; KLEIN, B.; BERGHETTI, M. R. P.; WENDT, L. M. Growth regulators on quality traits and volatile organic compounds profile of 'Royal Gala' apple at harvest and after dynamic controlled atmosphere storage. Postharvest Biology and Technology, v. 164, p. 1-12, 2020.

ANVISA. Resolução de Diretoria Colegiada - RDC № 07, de 18 de fevereiro de 2011. Ministério Da Saúde - MS Agência Nacional de Vigilância Sanitária - ANVISA, 2011.

BOTH, V.; BRACKMANN, A.; THEWES, F. R.; FERREIRA, D. D. F.; WAGNER, R. Effect of storage under extremely low oxygen on the volatile composition of "Royal Gala" apples. Food Chemistry, v. 156, p.50-57, 2014.

BOTH, V.; THEWES, F. R.; BRACKMANN, A.; DE OLIVEIRA ANESE, R.; DE FREITAS FERREIRA, D.; WAGNER, R. Effects of dynamic controlled atmosphere by respiratory quotient on some quality parameters and volatile profile of 'Royal Gala' apple after long-term storage. Food Chemistry, v. 215, p. 483-492, 2017.

BRACKMANN, A.; THEWES, F. R.; DE OLIVEIRA ANESE, R. ; BOTH, V.; JUNIOR, W. L.; SCHULTZ, E. E. Aminoethoxyvinylglycine: Isolated and combined with other growth regulators on quality of 'brookfield' apples after storage. Scientia Agricola, v. 72, n.3, p.221-228, 2015.

DIAS, J. V.; DA SILVA, R. C.; PIZZUTTI, I. R.; DOS SANTOS, I. D.; DASSI, M.;CARDOSO, C. D. Patulin in apple and apple juice: Method development, validation by liquid chromatography-tandem mass spectrometry and survey in Brazilian south supermarkets. Journal of Food Composition and Analysis, v.82, p.1-7, 2019.

FAO. Food and Agriculture Organization of the United Nations. http://www.fao.org/faostat/en/\#home/. Accessed 10.12.20. com baixo índice de podridão de todos os tratamentos, evidenciando, portanto, que o risco de fungos micotoxigênicos e micotoxinas é particularmente relevante para as indústrias de processamento de maçãs. CA forneceu a maior concentração de patulina $\left(166 \mu \mathrm{g} \mathrm{kg}^{-1}\right)$. As condições de armazenamento de DCA com menor concentração de $\mathrm{CO}_{2}$ foram associadas à menor concentração de patulina, sendo, portanto, uma melhor condição de armazenamento para esta cultivar.

FRISVAD, J. C.; SAMSON, R. A. Polyphasic taxonomy of Penicillium subgenus Penicillium: A guide to identification of food and air-borne terverticillate Penicillia and their mycotoxins. Studies in Mycology, v.49, p. 1-173, 2004.

OTEIZA, J. M.; KHANEGHAH, A. M.; CAMPAGNOLLO, F. B.; GRANATO, D.; MAHMOUDI, M. R.; SANT'ANA, A. S.; GIANUZZI, $\mathrm{L}$. Influence of production on the presence of patulin and ochratoxin A in fruit juices and wines of Argentina. LWT - Food Science and Technology, v. 80, p. 200-207, 2017.

PATRIARCA, A. Fungi and mycotoxin problems in the apple industry. Current Opinion in Food Science, v. 29, p. 42-47, 2019.

PITT, JOHN I.; HOCKING, A. D. Fungi and food spoilage. Fungi and Food Spoilage, p. 1-519, 2009.

PUEL, O.; GALTIER, P.; OSWALD, I. P. Biosynthesis and toxicological effects of patulin. Toxins, v. 2, n. 4, p. 613-631, 2010.

SAMSON R.A.; HOEKSTRA E.S.; FRISVAD J.C.; Introduction to Food and Airborne Fungi, v. 7. Centra al bureau voor Schimmel cultures, Utrecht, The Netherlands, 2004.

TANNOUS, J.; KELLER, N. P.; ATOUI, A.; EL KHOURY, A.; LTEIF, R.; OSWALD, I. P.; PUEL, O. Secondary metabolism in Penicillium expansum: Emphasis on recent advances in patulin research. Critical Reviews in Food Science and Nutrition, v. 58, n. 12, p. 2082-2098, 2018.

WATKINS, C. B. Storage Disorders of Controlled AtmosphereStored Empire Apples, v. 18, n.3, p. 19-22, 2010.

WEBER, A.; BRACKMANN, A.; BOTH, V.; PAVANELLO, E. P.; DE OLIVEIRA ANESE, R.; THEWES, F. R. Respiratory quotient: Innovative method for monitoring 'Royal Gala' apple storage in a dynamic controlled atmosphere. Scientia Agricola, v. 72, n. 1, p. 28-33, 2015. 